\title{
Diffusion as mixing mechanism in granular materials
}

\author{
C. Henrique ${ }^{1}$, G. Batrouni $^{2}$ and D. Bideau ${ }^{1}$ \\ ${ }^{1}$ Groupe Matière Condensée et Matériaux (UMR 6626), Université de Rennes I, Bât 11A, Campus de Beaulieu 35042 \\ Rennes Cedex, France \\ 2 Institut Non-Linéaire de Nice, Université de Nice-Sophia Antipolis, 1361 route des Lucioles, O6560 Valbonne, France
}

\begin{abstract}
We present several numerical results on granular mixtures. In particular, we examine the efficiency of diffusion as a mixing mechanism in these systems. The collisions are inelastic and to compensate the energy loss, we thermalize the grains by adding a random force. Starting with a segregated system, we show that uniform agitation (heating) leads to a uniform mixture of grains of different sizes. We define a characteristic mixing time, $\tau_{m i x}$, and study theoretically and numerically its dependence on other parameters like the density. We examine a model for bidisperse systems for which we can calculate some physical quantities. We also examine the effect of a temperature gradient and demonstrate the appearance of an expected segregation.
\end{abstract}

\section{INTRODUCTION}

Granular media are notoriously difficult to mix. For a variety of reasons and under rather general conditions, they tend to form segregated steady statest. For example, segregation can occur during granular flow, the bigger particles moving farther than the smaller ones. Difference of size (polydispersity of the grains) or difference of material (different kinds of grains) produce different geometrical or physical properties. Segregation can also be due to percolation where the small grains fall through the holes between the big grains leaving only the bigger particles behind. Shear $\mathbf{3}^{3}$ and vibration can also produce segregation. One of the best kngwen examples of vibration segregation is perhaps the "Brazil nut effect". In this case, the geometrical propertiesl g $_{\text {are }}$ responsable for the upward movement of big particles although convection processes near the boundaries can also be very important all these processes (flow, shearing and convection) are very common in industrial applications such as in mixers 10.11 . For these reasons such mixers are efficient only for rather homogeneous materials. In the polydisperse case it is very hard to avoid segregation.

For gases and liquids, the thermal agitation of molecules is a natural and efficient mechanism leading to throughly mixed systems with homogenous equilibrium steady states2. We propose here, a study of a system of agitated grains in analogy with liquid or gas molecules at the miroscopic scale.

Two major differences between granular materials and fluids are: (1) the particle size compared to the mean free path and (2) the inelastic properties and friction responsable for energy dissipation during collisions. The question then is if these differences will alter the system's natural tendency to mix by diffusion. In other words, is it possible to use diffusion to mix grains. In spite of the dissipative collisions, it is possible to keep a granular system agitated, for example on an air-table or a vibrating bed. To simulate numerically such constantly agitated granular systems, we add an external random force to the equations of motion (see section III). We then analyze the grain diffusion and its dependence on the various parameters of the system such as grain size. We find that inspite of the dissipative nature of the collisions, diffusion is still a good mixing mechanism just like in fluids.

In section II we detail the algorithm and summarize the principal dynamic equations and parameters of our system. We verify our procedure with the study of a monodisperse system in section III and etablish relations which characterize the temporal evolution of an initially segregated system. The bidisperse case is studied in section IV. We show in particular the evolution of a system with homogeneous agitation, and also the effects of a gradient in this agitation. Our conclusions and discussion are in section $\mathrm{V}$.

\section{ALGORITHM AND REVIEW}

We use an Event Driven Molecular Dynamics algorithm. The simulated orains have the same characteristics (e.g. normal and tangential restitution coefficients) as measured experimentally 13 . To thermalize the system, we add at regular time step intervals, $d t$, external random forces which act on every particle. There are several choices one can make for this force. Our choice is the following:

$$
F_{i}^{t}=m\left(\sqrt{\eta_{0}^{2} / d t}\right) \zeta_{i}
$$

with $i=x, y$ (corresponding to the two directions), $\zeta_{i}$ is a gaussian noise caracterized by $\left\langle\zeta_{i} \zeta_{j}\right\rangle=\delta_{i, j}$ and $m$ is the particle mass. $\eta_{0}^{2}$ is the control parameter which we use to increase or decrease the agitation. Experimentally, on the 
air table14, plastic disks of radius $R$ move due to the fluctuations of the air flux acting on their surfaces. Therefore, since their mass is proportional to $R^{2}$, we expect the acceleration to be independent of $R$. That is why we have chosen an external force proportional to the particle mass, Eq. (2.1). The equation of motion of a particle between two collisions becomes:

$$
\frac{d \mathrm{v}_{\mathrm{i}}}{d t}=\sqrt{\eta_{0}^{2}} \zeta_{i}
$$

In this paper $\mathrm{v}_{\mathrm{i}}$ denotes the instantaneous velocity in the $\mathrm{i}$ direction and $v^{2}$ the mean square velocity. The system is two dimensional and is enclosed in a square box whose walls are made of grains of radius $r_{w}$ and are infinitely massive. The particle-wall collisions are taken to be elastic. Note that in these two-dimensional simulations, the particles are represented as spheres interacting at their equators.

\section{A. Macroscopic characteristics of the steady state}

The above model leads to a steady state characterized by a constant mean square velocity, $v^{2}(t \rightarrow \infty)$ for all particles. The energy loss during collisions is compensated for by the random force. For a monofisperse gas, the energy balance is easily calculated. The energy loss, $\Gamma$, per unit time in the steady state is given by 15

$$
\Gamma \propto \omega m v^{2}
$$

where $\omega$ is the frequency of collisions. On the other hand, the average gain in energy due to the random force during $d t$ is easily obtained from Eq. (2.2):

$$
\frac{1}{2} m\left[v^{2}(t+d t)-v^{2}(t)\right]=m \eta_{0}^{2} d t
$$

In the steady state we can thus write

$$
\frac{1}{2} m \frac{\partial v^{2}}{\partial t}=-\Gamma+m \eta_{0}^{2} .
$$

We can write $\omega \sim \frac{\sqrt{v^{2}}}{l}$ where $l$ is a caracteristic length depending only on the packing fraction and on the radius of the grains. With this assumption and the fact that $\frac{\partial v^{2}(t \rightarrow \infty)}{\partial t}=0$, Eq. (2.5) gives

$$
v^{2}(\infty) \propto\left(l \eta_{0}^{2}\right)^{2 / 3}
$$

This power law is independent of the coefficients of restitution and friction if dissipation is not too large. The results of the kinetic theory of inelastic gases can be applied, in particular the velocity distribution can be approximated by a Maxwellian. The parameter $\eta_{0}^{2}$ allows us to change the granular temperature, $T$, since $v^{2} \propto T$. Therefore, in the steady state, $T$ is independent of the initial conditions. For polydisperse gases the problem becomes more complicated as will be seen below.

\section{B. Coefficient of diffusion}

Since the mean square velocity is constant, and consequently the collision frequencies too, particles have a simple diffusive behavior. The mean square displacement, $\left\langle\left(r\left(t+t_{0}\right)-r\left(t_{0}\right)\right)^{2}\right\rangle$, gives the coefficient of diffusion

$$
<\left(r\left(t+t_{0}\right)-r\left(t_{0}\right)\right)^{2}>=4 D t
$$

where $t_{0}$ is large enough to ensure thermalization of the system. Clearly, if the system is examined at a time scale $t \leq 1 / \omega$ we will not observe the true diffusive behaviour. At short time, $\mathrm{v}_{\mathrm{i}}$ is not constant due to the action of $F_{i}^{t}$ and so the mean square displacement is not yet linear with $t$. For $t>>1 / \omega$ we verify the linear dependence of the mean square displacement on time, for all particles. The value of the coefficient of diffusion $D$ found from the simulation is thus larger than the theoretical value predicted by a Langevin description due to the dynamics of the particle at short time 16 . 


\section{Simulation procedure}

We study systems made of two species of grains, $s$ and $b$. The radius of the particles are respectively $R_{s}$ and $R_{b}$. The system is a square box of length $L$ and we use the boundary conditions discussed above. The number of particles of each species is calculated based on the desired packing fraction $C$ and the relative proportion of $s$ particles $x_{s}$.

$$
\left\{\begin{array}{l}
C=\frac{n_{s} \pi R_{s}^{2}+n_{b} \pi R_{b}^{2}}{L^{2}}, \\
x_{s}=\frac{n_{s} \pi R_{s}^{2}}{n_{s} \pi R_{s}^{2}+n_{b} \pi R_{b}^{2}},
\end{array}\right.
$$

where $n_{s}$ and $n_{b}$ are repectively the number of particles $s$ and $b$.

For all mixtures, we performed two types of simulations. In the first one, the two species $s$ and $b$ are already mixed and the initial position of each particle is chosen randomly in the box by using a classical algorithm of Random Sequential Adsorption (R. S. A.) 18. We are careful that this algorithm does not introduce segregation in the initial configuration. For the second type of simulation, the two species are initially separated with the $s$ particles on the right and the $b$ particles on the left. The system is prepared such that the packing fraction is homogeneous in the whole system.

In the following section we present our results in the simple case where the two species have the same mechanical and geometrical properties, i.e. $s$ and $b$ grains are of the same type.

\section{MONODISPERSE CASE}

To test the validity of our algorithm we start with identical grains, i.e. $R_{s}=R_{b}$. In this case, we do not expect segregation because the grains are identical, but we would like to verify that the thermal process is efficient and that particles do not agregate. In other words, after some time each grain will have visited all regions of the box. In figure 11 is presented the temporal evolution of the system for the two different initial configurations specified above. In figure 1 1 a the system is already mixed and in $1 \mathbf{b}$ the particles are initially separated. As one can see in this figure, the system does not collapse and the grains are homogeneously distributed in the box. To analyse the dynamics of the mixture we measure the quantity $N_{s, b}(t)$ defined as the number of collisions between $s$ and $b$ grains per unit time. The evolution of $N_{s, b}(t)$ with time gives two important results. For the initial configuration corresponding to figure 1 a, the quantity $N_{s, b}(t)$ fluctuates around a mean value, $N_{s, b}(\infty)$, as seen in figure 2. This means that the system has reached a steady state in which the mean square velocity of the particles is constant and equal to $v^{2}(\infty)$. Note that the system evolves very quickly into this steady state. We have checked that all the configurations at different times, $t$, are statistically identical and that the system remains homogeneous. There is no evidence of collapse or cluster formation. The second observation is that for the initial configuration corresponding to figure $\mathbb{b} \mathbf{b}$, the quantity $N_{s, b}(t)$ increases and then stabilizes at large time at the value $N_{s, b}(\infty)$ defined above. However, the mean square velocity of the grains, $v^{2}(t)$, reaches the steady state value $v^{2}(\infty)$ much more quickly since the grains are identical. The knowledge of the mean square velocity is not sufficient to define the state of the system since it gives no information about the spatial repartition of the two species. $N_{s, b}(t)$ is therefore the only pertinent quantity to characterize the homogeneity of the system.

A few more comments about $N_{s, b}(t)$ are in order. In the system studied above, the packing fraction and the velocity distribution are spatially homogeneous and constant in time (except at very short time). As a consequence the quantity $N_{s, b}(t)$ depends only on the spatial repartition of the two types of grains. The evolution of $N_{s, b}(t)$ allows us to define a mixing time, $\tau_{m i x}$. We have already mentioned that the velocity is the same for all particles and independent of position. This is also true for the local density. We conclude that also the frequency of collisions is the same for all particles and is space independent. In this monodisperse case, the dynamics are purely diffusive and can be characterized by a coefficient of diffusion $D$ which is independent of horizontal spatial position $x$. Let us call $\delta N_{s, b}(x, t)$ the number of collisions between the two species occuring at a position between $x$ and $x+d x$ at time $t$. Clearly, $\delta N_{s, b}(x, t)$ is directly proportionnal to $d_{s}(x, t)$ and $d_{b}(x, t)$, the densities of $s$ and $b$ grains at position $x$. The densities $d_{s}$ and $d_{b}$ do not depend on the vertical position since the system is invariant along this direction. We will define $d_{0}$ as the total density and can, thus, write $d_{0}=d_{s}(x, t)+d_{b}(x, t)$. $d_{0}$ is of course independent of $x$ because the system remains homogeneous. We then obtain an expression for $N_{s, b}(t)$ :

$$
N_{s, b}(t) \propto \int_{0}^{L} d_{b}(x, t)\left(d_{0}-d_{b}(x, t)\right) d x .
$$


The density of big particles at $(x, t), d_{b}(x, t)$, is described by Fick's equation,

$$
\frac{\partial d_{b}(x, t)}{\partial t}=D \frac{\partial^{2} d_{b}(x, t)}{\partial x^{2}}
$$

with the following boundary conditions:

$$
\begin{cases}d_{b}(x)=d_{0} & \text { for } 0 \leq x<L / 2 \text { and } t=0 \\ d_{b}(x)=0 & \text { for } L / 2 \leq x \leq L \text { and } t=0, \\ d_{b}(x)=d_{0} / 2 & \text { for all } x \text { and } t \simeq \infty\end{cases}
$$

We assume the solution of Eq. (3.2) has the form:

$$
d_{b}(x, t)=\sum_{m=0}^{\infty}\left(B_{m} \sin \left(\lambda_{m} x\right)+A_{m} \cos \left(\lambda_{m} x\right)\right) \exp \left(-\lambda_{m}^{2} D t\right)+\frac{d_{0}}{2}
$$

where the $\lambda_{m}$ are constants. Using the conditions Eq. (3.3) gives:

$$
d_{b}(x, t)=\sum_{k=0}^{\infty} a_{k} \cos \left(\lambda_{k} x\right) \exp \left(-\lambda_{k}^{2} D t\right)+\frac{d_{0}}{2}
$$

with

$$
\begin{aligned}
a_{k} & =\frac{2 d_{0}(-1)^{-k}}{\pi(2 k+1)}, \\
\lambda_{k} & =\frac{(2 k+1) \pi}{L} .
\end{aligned}
$$

Eq. (3.1) then gives the final expression for $N_{s, b}(t)$,

$$
\begin{gathered}
N_{s, b}(t) \propto \frac{d_{0}^{2} L}{4}\left(1-\sum_{k=0}^{\infty} \frac{8}{\pi^{2}(2 k+1)^{2}} \exp \left(-2 \lambda_{k}^{2} D t\right)\right), \\
\lambda_{k}^{2}=\frac{(2 k+1)^{2} \pi^{2}}{L^{2}} .
\end{gathered}
$$

As a first approximation, we may keep only the first mode and write:

$$
\begin{gathered}
N_{s, b}(t) \simeq N_{s, b}(\infty)\left(1-\exp \left(-t / \tau_{\text {mix }}\right)\right), \\
\tau_{\text {mix }}=\frac{L^{2}}{2 \pi^{2} D},
\end{gathered}
$$

where $\tau_{m i x}$ can be taken as the typical time for mixing.

To check the validity of the theoretical expressions for $N_{s, b}(t)$ and $\tau_{m i x}$ established above, we have performed simulations for different values of $x_{s}$ and $C$. For a given set of parameters, we have performed five simulations corresponding to different initial positions and velocities of the particles for the case where the $s$ and $b$ grains are initially separated. Figure 3 shows $N_{s, b}(t)$ versus $t$ averaged over the 5 simulations. We see that the agreement between theory and numerical simulation is very good. Figure 4 shows the dependence of the mixing time, $\tau_{m i x}$, on the coefficient of diffusion $D$. To get this, we performed several simulations changing the packing fraction and the radius of the particles in order to vary the coefficient of diffusion. Note that $D$ was estimated using Eq. (2.7). The slope of the curve is exactly that predicted by the theory. As the coefficient $D$ can be calculated from the parameters of the system $\left(R_{s}=R_{b}, \eta_{0}^{2}, C\right)$, we can estimate analytically and with high accurancy the mixing time. We have also verified the dependence of $\tau_{m i x}$ on $L^{2}$, Eq. (3.8), and have found very good agreement too.

\section{BIDISPERSE CASE}

We now discuss the case of a binary mixture. We will see that the size difference between the grains changes drastically the dynamics of the system. The grains $s$ and $b$ are taken of equal density and identical coefficients of restitution and friction, and we take $R_{s}<R_{b}$.

We present first the case where the system is thermalyzed uniformly, i.e. $F_{i}^{t}$ does not depend of the position of the grain. Then we will examine the case where a gradient is imposed on the agitation force. 


\section{A. Case of homogeneous agitation}

We will show that, in the bidisperse case, the system also evolves into a homogeneous steady state. We will see here that the form of the thermalization force and the initial conditions determine the evolution of the system towards the steady state. In the simulations the packing fraction is fixed to $40 \%$ and $x_{s}$, which represents the relative proportion of small grains (Eq. (2.8)), is the only parameter to be varied.

\section{Evolution at short time}

Figure 5 shows the evolution of the system with time $t$. In the initial configuration, the two species are separated and the two populations have the same initial velocity distribution and therefore $v_{s}^{2}=v_{b}^{2}$. The initial local packing fraction, as one can see in figure 5, is the same in the whole box.

Recall that in our simulations the surface occupied by a particle in the plane is proprotional to $R^{2}$ and its mass to $R^{3}$ since the particles are spherical. Since the pressure is $P \propto m d v^{2}$, where $d$ is the density of grains, the initial pressure is larger for the bigger particles. The system therefore has an initial pressure difference which will govern its behaviour immediately after the partition is removed whereby the bigger particles, $b$, compress the small ones $s$. As $t$ increases, (see figure 5) the density of the $b$ particles decreases and so does its pressure. On the other hand, the density and pressure of the $s$ particles increase. During this compression period we can consider the system as two interacting monodisperse systems. In the left part of the box (occupied by the larger particles), as the density decreases, the mean free path, $l$ increases. We have seen in section II A (see eq 2.6) that $v^{2}$ increases with $l$. The velocity $v_{b}^{2}$ is then increasing with time. For the same reason, in the right part of the box, $v_{s}^{2}$ is decreasing. As a consequence, the pressure, which is proportional to the product of the square velocity and the density, is maintained almost constant in each subsystem. The pressure difference between the two subsystems remains therefore important and favors the compression of small particles. The packing fraction of the $s$ grains increases up to a value around $68 \%$.

It is worth noting that if the walls were inelastic, collapse would occur whereby the small grains would be squeezed near the wall and would loose all their energy due to dissipation. In our simulations we use elastic wall and thus observe a reflection of the compression wave. To illustrate this we show in figure 6 the packing fraction of the $s$ grains as a function of $t$ and $x$. We observe a compression wave which traverses the system. On average, the small particles remain compressed and the big ones dilute. During this process the diffusion between the big and the small particles is very efficient due to the high concentration gradient. The evolution of the quantities $v_{s}^{2}(t)$ and $v_{b}^{2}(t)$ as a function of time is illustrated in figure [7. One can see that the velocity of small particles decreases at short time and then increases when the mixing process starts. At long time the mean square velocities of both species reach a constant value corresponding to a steady state.

\section{Mixing time}

After the compression phase, the system starts to mix. As we have done for the monodiperse case we examine the quantity $N_{s, b}(t)$. To have a good estimate of $N_{s, b}(t)$, we take (as in the previous section) the mean value obtained over 5 simulations. Figure 8 shows that we can appoximate $N_{s, b}$ by $N(\infty) \exp \left(-t / \tau_{m i x}\right)$. Note that the compression phase occurs during a short time compared to $\tau_{m i x}$. We obtain in this way $\tau_{m i x}$ for different values of $x_{s}$ for $R_{s}=0.4$ and $R_{b}=0.6$. It appears that this time $\tau_{m i x}$ can be considered as independent of $x_{s}$ (see Figure 9). This mixing time obtained in a bidisperse system is smaller than that obtained for the same packing fraction in the monodisperse case.

\section{Steady state}

Even though the collisions are dissipative, the system does reach an out of equilibrium stationary state due to the random agitation force. This stationary state should be characterized by macroscopic functions which should be independent of time.

Using thermalized configurations (long evolution times), we performed a geometrical analysis using Voronoï tessellation to check that no segregation exists. We calculated the number of $s$ neighbours for a $b$ particle and found that the distributions of neighbours are roughly identical to the distributions obtained from static configurations generated by an R.S.A. algorithm. We should point out that the distances between particles can be different from the static 
case but the neighbourhood of a grain is the same in the dynamic and static situations. This demonstrates that there is no segregation.

We now consider the distribution of the kinetic energy as a function of the radius of grains. In the case of elastic collisions, we can define a kinetic temperature $T$ even in a polydisperse case. In forced inelastic system the repartition of energy seems to be very different and depends on the type of forcing used 1920.

In our system, the mean square velocity $v_{i}^{2}$ of particle $i$ depends on its mass $m_{i}$ and also on the proportion of all species $j$ and their masses $m_{j}$. In all cases $v_{i}^{2}$ is constant at large time. Energy balance in a bidisperse system means that the agitation energy per unit time for a given species equals the energy lost in collisions with particles from all species. This can be written as follows:

$$
\left\{\begin{array}{l}
P\left(m_{s}, m_{s}\right) w_{s s} m_{s} v_{s}^{2}+P\left(m_{s}, m_{b}\right) w_{s b} m_{s} v_{s}^{2}=m_{s} \eta_{0}^{2} \\
P\left(m_{b}, m_{b}\right) w_{b b} m_{b} v_{b}^{2}+P\left(m_{b}, m_{s}\right) w_{b s} m_{b} v_{b}^{2}=m_{b} \eta_{0}^{2}
\end{array}\right.
$$

where $P\left(m_{i}, m_{j}\right)$ is the mean relative loss of kinetic energy by particle $i$ when particles $i$ and $j$ collide. Clearly, this term depends both on mass and relative velocity. We also expect that $P\left(m_{s}, m_{s}\right)$ should be equal to $P\left(m_{b}, m_{b}\right)$. Therefore the quantities $P\left(m_{s}, m_{s}\right)$ and $P\left(m_{b}, m_{b}\right)$ calculated in a bidisperse system must be the same as those obtained for the monodisperse case. This term $P\left(m_{i}, m_{i}\right)$ is therefore only a function of coefficients of restitution and friction. The term $w_{i}$ represents the frequency of collisions of the grains $i$ with $j$ grains. We note that $n_{s} w_{s b}=n_{b} w_{b s}$. Using Enskog theory 21 , the frequencies of collisions of the grains in our two-dimensional system are given by:

$$
\left\{\begin{array}{l}
w_{s s}=\chi \sqrt{\pi}\left(2 R_{s}\right) \frac{C x_{s}}{\pi R_{s}^{2}} \sqrt{2 v_{s}^{2}} \\
w_{s b}=\chi \sqrt{\pi}\left(R_{s}+R_{b}\right) \frac{C\left(1-x_{s}\right)}{\pi R_{b}^{2}} \sqrt{v_{s}^{2}+v_{b}^{2}} \\
w_{b b}=\chi \sqrt{\pi}\left(2 R_{b}\right) \frac{C\left(1-x_{s}\right)}{\pi R_{b}^{2}} \sqrt{2 v_{b}^{2}} \\
w_{b s}=\chi \sqrt{\pi}\left(R_{s}+R_{b}\right) \frac{C x_{s}}{\pi R_{s}^{2}} \sqrt{v_{s}^{2}+v_{b}^{2}}
\end{array}\right.
$$

$\chi$ is a correction factor and corresponds to the local radial distribution around a particle22 23. We have previously shown in 24 that $\chi$ does not depend on the type of particles but only on the packing fraction.

The two limit cases, $x_{s}=0$ and $x_{s}=1$, correspond to monodisperse situations with $R=R_{s}$ and $R=R_{b}$ respectively. In these two cases, we have determined numerically the four parameters $v_{s}^{2}\left(x_{s}=0\right), v_{s}^{2}\left(x_{s}=1\right)$, $v_{b}^{2}\left(x_{s}=0\right)$ and $v_{b}^{2}\left(x_{s}=1\right)$ by simulating a particle of radius $R_{i}$ in a sea of particles of radius $R_{j}$. Using Eq. (4.1), we can calculate for these limiting values of $x_{s}$ the four parameters $P\left(m_{i}, m_{j}\right)$. We have verified that $P\left(m_{i}, m_{i}\right)$ is independent of the type of particle. We have found that $P\left(m_{s}, m_{s}\right)=P\left(m_{b}, m_{b}\right)=0.145, P\left(m_{b}, m_{s}\right)=0.229($ at $x_{s}=1$ ) and $P\left(m_{s}, m_{b}\right)=0.066\left(\right.$ at $\left.x_{s}=0\right)$.

To a first approximation, we consider the $P\left(m_{i}, m_{j}\right)$ to be independent of the relative velocity. We compare in figure 10 the values of $v_{s}^{2}$ and $v_{b}^{2}$ obtained from the numerical simulations and those deduced from Eq. (4.1). These values were calculated for different $x_{s}$ at a packing fraction of $40 \%$. The dashed lines correspond to the theoretical values and the symbols to the simulations. The agreement between simulations and theory is quite good, in particular for the big particles.

However, the energy lost in a collision does depend on the relative velocity, and therefore on $v_{s}^{2}$ and $v_{b}^{2}$. To treat this correctly in Eq. (4.1), we show in figure 11 that both, the kinetic energy of the system and $v_{s}^{2} / v_{b}^{2}$, decrease linearly with $x_{s}$. Recalling that $x_{s}$ lies in the interval $[0,1]$, we can thus write:

$$
\left\{\begin{array}{l}
\frac{C x_{s}}{\pi R_{s}^{2}} m_{s} v_{s}^{2}\left(x_{s}\right)+\frac{C\left(1-x_{s}\right)}{\pi R_{b}^{2}} m_{b} v_{b}^{2}\left(x_{s}\right)=\frac{C}{\pi R_{b}^{2}} m_{b} v_{b}^{2}\left(x_{s}=0\right)+\left[\frac{C}{\pi R_{s}^{2}} m_{s} v_{s}^{2}\left(x_{s}=1\right)-\frac{C}{\pi R_{b}^{2}} m_{b} v_{b}^{2}\left(x_{s}=0\right)\right] x_{s}, \\
\frac{v_{s}^{2}\left(x_{s}\right)}{v_{b}^{2}\left(x_{s}\right)}=\frac{v_{s}^{2}\left(x_{s}=0\right)}{v_{b}^{2}\left(x_{s}=0\right)}+\left[\frac{v_{s}^{2}\left(x_{s}=1\right)}{v_{b}^{2}\left(x_{s}=1\right)}-\frac{v_{s}^{2}\left(x_{s}=0\right)}{v_{b}^{2}\left(x_{s}=0\right)}\right] x_{s} .
\end{array}\right.
$$

Using the four equations 4.3 and 4.1, we can calculate directly $v_{s}^{2}, v_{b}^{2}, P\left(m_{s}, m_{b}\right)$ and $P\left(m_{b}, m_{s}\right)$ as a function of $x_{s}$. Note that different values of $x_{s}$ correspond to different values of the ratio $v_{s}^{2} / v_{b}^{2}$. The solid lines in figure 10 correspond to the theoretical velocities squared calculated with this approach. The agreement with simulations is now very good. The values of $P\left(m_{s}, m_{b}\right)$ and $P\left(m_{b}, m_{s}\right)$ from the solution of our four equations are shown in figure 12. The values of $P\left(m_{s}, m_{b}\right)$ for $x_{s}$ near 1 and $P\left(m_{b}, m_{s}\right)$ for $x_{s}$ near 0 should be taken with precaution, because their weight in the balance of energy (Eq. (4.1)) is negligeable for $x_{s} \simeq 1$ and $x_{s} \simeq 0$. Indeed the energy loss in these limit cases corresponds to rare collisions.

Note that the approximation of $P\left(m_{b}, m_{s}\right)$ by a constant (as done in the first approach) is fairly good. However, the value of $P\left(m_{s}, m_{b}\right)$ varies significantly with $x_{s}$. This explains the slight difference between simulation and theory in the first approach. Finally we see that at large $x_{s}$ (corresponding to small ratio $v_{s}^{2} / v_{b}^{2}$ ) the small particles gain energy in collisions with big particles. This phenomenon occurs only in dissipative forced gases where $v^{2}$ is no longer proportional to $1 / \mathrm{m}$. 


\section{B. Non-uniform agitation}

We will now treat the case of non-uniform agitation. To do this we use exactly the same algorithm but introduce a gradient in the agitation by imposing the following spatial dependence for $\eta_{0}^{2}$ :

$$
\eta_{0}^{2}(x)=a+b x
$$

where $a$ and $b$ are constants. The initial configuration of the system is taken to be the stationary state found in the case of homogeneous agitation. The gradient of the agitation is chosen such that the mean value of $\eta_{0}^{2}(x)$ over the whole system corresponds to the agitation of the initial state. The simulations show that a concentration gradient appears in the system. The system reaches a non-uniform steady state where the density gradient remains present in the course of time. Note, however, that if we follow the motion of a particle, we find that it does visit the whole system. All the previous relations and equations are still valid in this case but one should consider a local "equilibrium". Figure 13 represents a typical configuration obtained at large time. The hot agitation is on the right side of the system. In the stationary state, the gradient of concentration balances the gradient of agitation such that the pressure is homogeneous throughout the system.

The other main observation is that segregation appears in the system. The local proportion of $s$ and $b$ grains is no longer the initial one. The big particles are more sensitive to the gradient of agitation than the small ones. We show in figure 14 the local packing fraction for both species as a function of the position $x$ in the system. We have found in all cases we have investigated so far that the stationary state always exhibits segregation. Our numerical results on segregation (the big particles are more concentrated in the colder region) is in complete agreement with the theoretical calculations based on the granular kinetic theoryes.

\section{CONCLUSION}

The main purpose of this paper is to investigate granular mixtures numerically. We used an algorithm which keeps the dissipative particles agitated by applying to the grains an external random acceleration independent of their mass. In the case where the external acceleration is independent of the position of the particles (homogeneous agitation) we have shown that the system reaches a well mixed homogeneous stationary state even in the bidisperse case. We have therefore shown that agitation, and therefore diffusion, is an efficient mechanism for mixing. We have established a theoretical expression between the typical time of mixing, $\tau_{m i x}$, and the number $N_{s, b}$ of collisions between $s$ and $b$ grains per unit of time which is valid for monodisperse as well as bidisperse systems. For the monodiperse case we have given the exact expression of $\tau_{m i x}$ as a function of the diffusion coefficient. For the bisperse case we have found that $\tau_{\text {mix }}$ depends strongly on the initial configuration of the system. We have characterized the steady state reached by bidisperse assemblies and in particular we have established energetic relations which allow us to evaluate the square velocities of the grains as a function $x_{s}$ (the relative proportion of both species).

In addition we have investigated the case where there is a gradient of agitation through the system and have shown that segregation appears. The main cause of this segregation is not related directly to the nature of the grains (size, dissipation, roughness) but originates from the presence of a temperature gradient. In a lot of mixing experiments (rotating drum, vibrated system) there often exists a gradient of agitation which then leads to a segregation process. Of course there are other types of segregation mechanisms which are sometimes more efficient, but this one is in some sense intrinsic.

\section{Acknowledgments}

This work was partially funded by the CNRS Programme International de Cooperation Scientifique PICS \#753. C. H. thanks Alexandre Valance for his support during this work and his help for writing of this paper.

\footnotetext{
${ }^{1}$ E. Guyon and D. Bideau in Proc. of the workshop "Instabilities and Non Equilibrium Structures" Ed. E. Tirapegui (1996)

${ }^{2}$ L. Samson, PhD thesis Université de Rennes (1997)

${ }^{3}$ A. M. Scott and J. Bridgwater, Powd. Tech. 14 (1976) 177-183

${ }^{4}$ C. S. Cambell Ann. Rev. Fluids. Mech. 22 (1990) 57-92
} 
${ }^{5}$ S. B. Savage and C. K. K. Lun, J. Fluid Mech. 189 (1998) 311-335

${ }^{6}$ G. H. Rosato, K. J. Strandburg, F. Prinz and R. H Swendsen; Phys. Rev. Lett. 58 (1997) 1038-1040

${ }^{7}$ R. Julien, P. Meakin and A. Pavlovitch, Phys. Rev. Lett 69 (1992) 640-643

${ }^{8}$ J. Duran, T. Mazozi, S. Luding, E. Clément and J. Rajchenbach, Phys. Rev. E 53, 1923 (1996)

${ }^{9}$ J. B. Knight, H. M. Jaegger and S. Nagel Phys. Rev. Lett. 70 (1993) 3728-3731

${ }^{10}$ P. M. C. Lacey, J. Appl. Chem. 4 (1954) 257

${ }^{11}$ L. T. Fan and Y. M. Chen, Powd. Tech. 61 (1990) 255-287

${ }^{12}$ E. Guyon and J. P. Hulin, "Granites et fumes un peu d'ordre dans le mélange" (Odile Jacob 1997)

13 M. Y. Louge, Phys. Fluids A, 6 (1994) 2253

14 J. Lemaitre, A. Gervois, H. Peerhossaini, D. Bideau and J. P. Troadec J. Phys. D 23 (1990) 1396

15 T. P. C. Van Noije, M. H. Ernst, E. Trizac, Phys. Rev. E 59 (1999) 4326

${ }^{16}$ C. Henrique, G. G. Batrouni, D. Bideau, "Effect of boundary conditions on diffusion in two-granular gases" Phys. Rev. E (submitted)

${ }^{17}$ C. Henrique, G. G. Batrouni, D. Bideau, Granular gases Worshop

18 J. Talbot and P. Schaaf Phys. Rev. A 40 (1989) 422-427

${ }^{19}$ I. Ippolito, C. Annic, J. Lemaitre, L. Oger, and D. Bideau, Phys. Rev. E 52 (1992)

${ }^{20}$ Y. Limon Duparcmeur, H. Herrmann and J. P. Troadec, J. Phys. I 5 (1995) 1119-1128

${ }^{21}$ S. Chapman and T. G. Cowling "The mathematical theory of non-uniform gases" (Cambridge University Press 1970)

${ }^{22}$ J. T. Jenkins and S. B. Savage J. Fluids. Mech. 130 (1983) 187-202

${ }^{23}$ Verlet and Levesque. Molecular Phys. 46 (1982) 969-980

${ }^{24}$ C. Henrique, PhD thesis Université de Rennes 1. (1999)

${ }^{25}$ B. Ö. Anarson and J. T. Willis, Phys. Fluids, 10 (1998) 1324

FIG. 1. Configurations at three different times $t=1, t=50$ and $t=199$. a): for an initially mixed system and $\mathbf{b}$ ): for an initially segregated system.

FIG. 2. Number of collisions per unit time between the two species as a function of time. The dashed (solid) line corresponds to the case of FIG. 1 a (b).

FIG. 3. $N_{s, b}(\infty)-N_{s, b}(t)$ vs time. Solid line is the numerical average over 5 simulations. The dashed line is given by $N_{s, b}(\infty) \exp \left(-t / \tau_{m i x}\right), \tau_{m i x}=43.5$.

FIG. 4. Dependence of $\tau_{m i x}$ on $D$. $\bigcirc$ : value from simulations. Solid line: theoretical prediction.

FIG. 5. Evolution, at short time, of an initially segregated bidisperse system; From left to right $t=1, t=6$ and $t=36$. $R_{s}=0.4$ and; $R_{b}=0.6$, the total packing fraction is $40 \% . x_{s}=0.5$.

FIG. 6. The local packing fraction of the small particles as a function of $x$ (the lateral position) and $t$.

FIG. 7. $v_{s}^{2}(t)$ and $v_{b}^{2}(t)$ as functions of time, for an initially segregated system.

FIG. 8. $N_{s, b}(t)$ vs $t$ for a bidisperse gas. $R_{s}=0.4, R_{b}=0.6$, and $x_{s}=0.625 . \tau_{m i x} \simeq 30$ is obtained by fitting Eq. (3.8), shown as a dashed line.

FIG. 9. $\tau_{m i x}$ for the bidisperse case as a function of $x_{s} . R_{s}=0.4, R_{b}=0.6$ and $C=0.4$. $\tau_{m i x}$ is constant.

FIG. 10. The mean square velocities reached in the stationary state as a function of $x_{s} . v_{s}^{2}(\square)$ and $v_{b}^{2}(\bigcirc)$ obtained from simulations. Dashed lines are obtained from eq 4.1 assuming constant $P\left(m_{i}, m_{j}\right)$ 's. Solid lines are the results from Eq. (4.1) and Eq. 4.3. 
FIG. 11. a) The total kinetic energy of the system as a function of $x_{s}$ for $R_{s}=0.4, R_{b}=0.6, C=0.4$ and $\eta_{0}^{2}=22.0$. b) $v_{s}^{2} / v_{b}^{2}$ as a function of $x_{s}$ for the same parameters.

FIG. 12. Values of $P\left(m_{s}, m_{b}\right)$ and $P\left(m_{b}, m_{s}\right)$ vs $x_{s}$ calculated using eqs 4.3 and 4.1 for the same mixtures as in FIG. 11 Dashed line: $P\left(m_{b}, m_{s}\right)$. Full line: $P\left(m_{s}, m_{b}\right)$.

FIG. 13. Typical configuration obtained in the presence of a gradient in the granular temperature. $R_{s}=0.4, R_{b}=0.6$, $x_{s}=0.5, a=4$ and $b=0.9$. $C=0.3$.

FIG. 14. Local packing fraction as a function of $x$ for $s(\square)$ and $b(\bigcirc)$ grains. The mixture corresponds to FIG. 13. Averaged over the whole system, $x_{s}=0.5$ and $C=0.3$. 

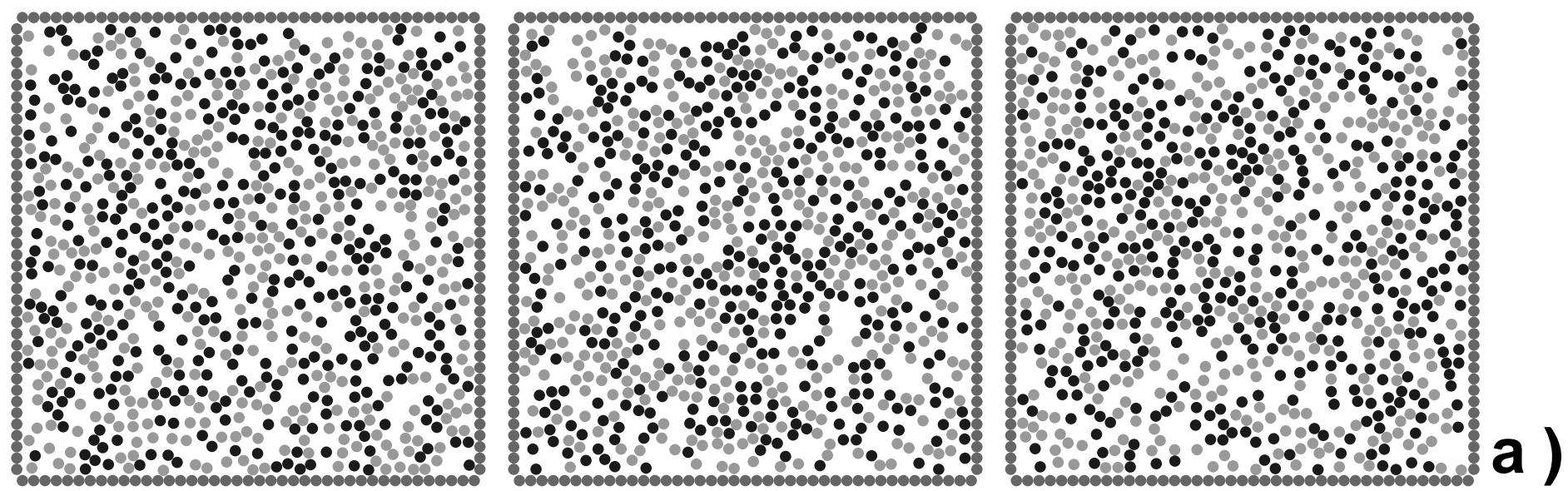

Figure 1.a

C. Henrique et. al.

"Diffusion as mixing process in granular materials" 

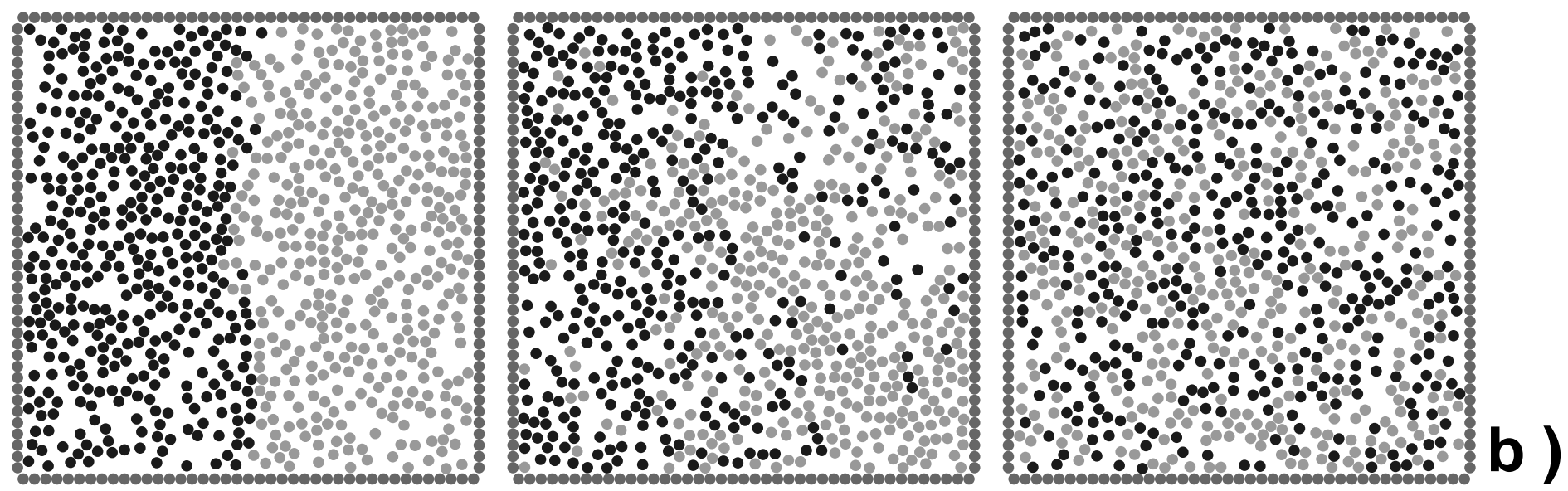

Figure 1.b

C. Henrique et. al.

"Diffusion as mixing process in granular materials", 


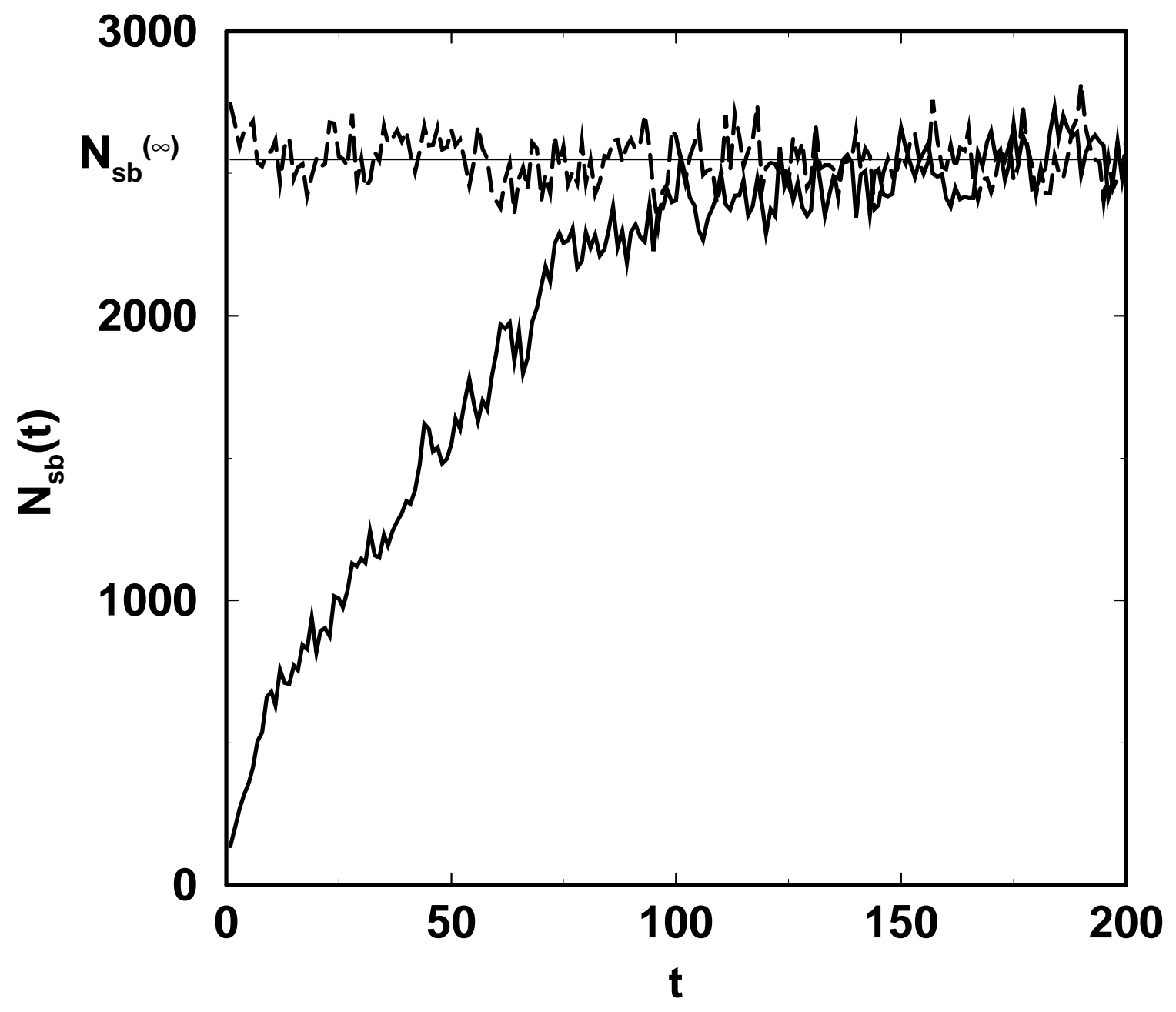

Figure 2

C. Henrique et. al.

"Diffusion as mixing process in granular materials", 


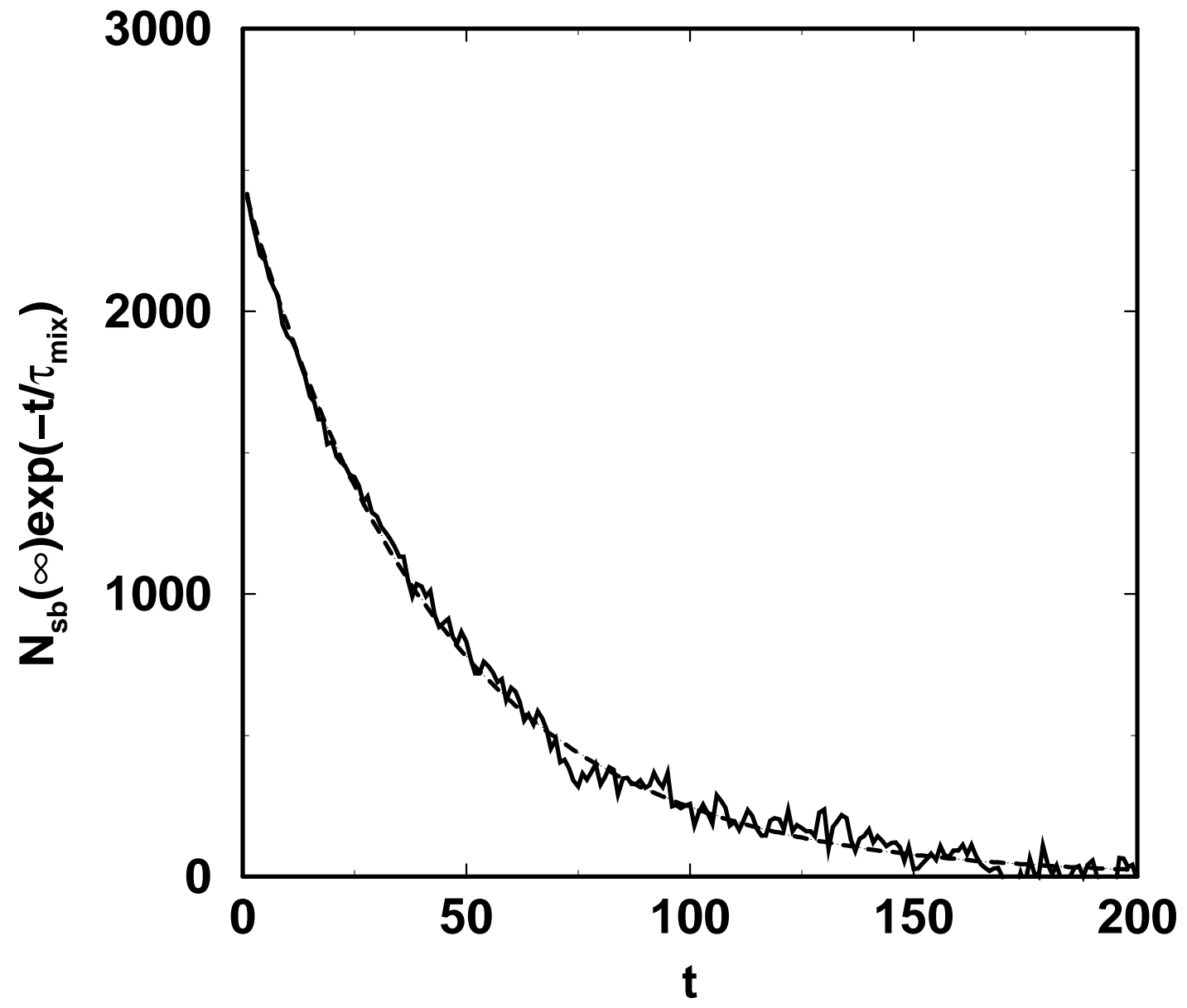

Figure 3

C. Henrique et. al.

"Diffusion as mixing process in granular materials", 


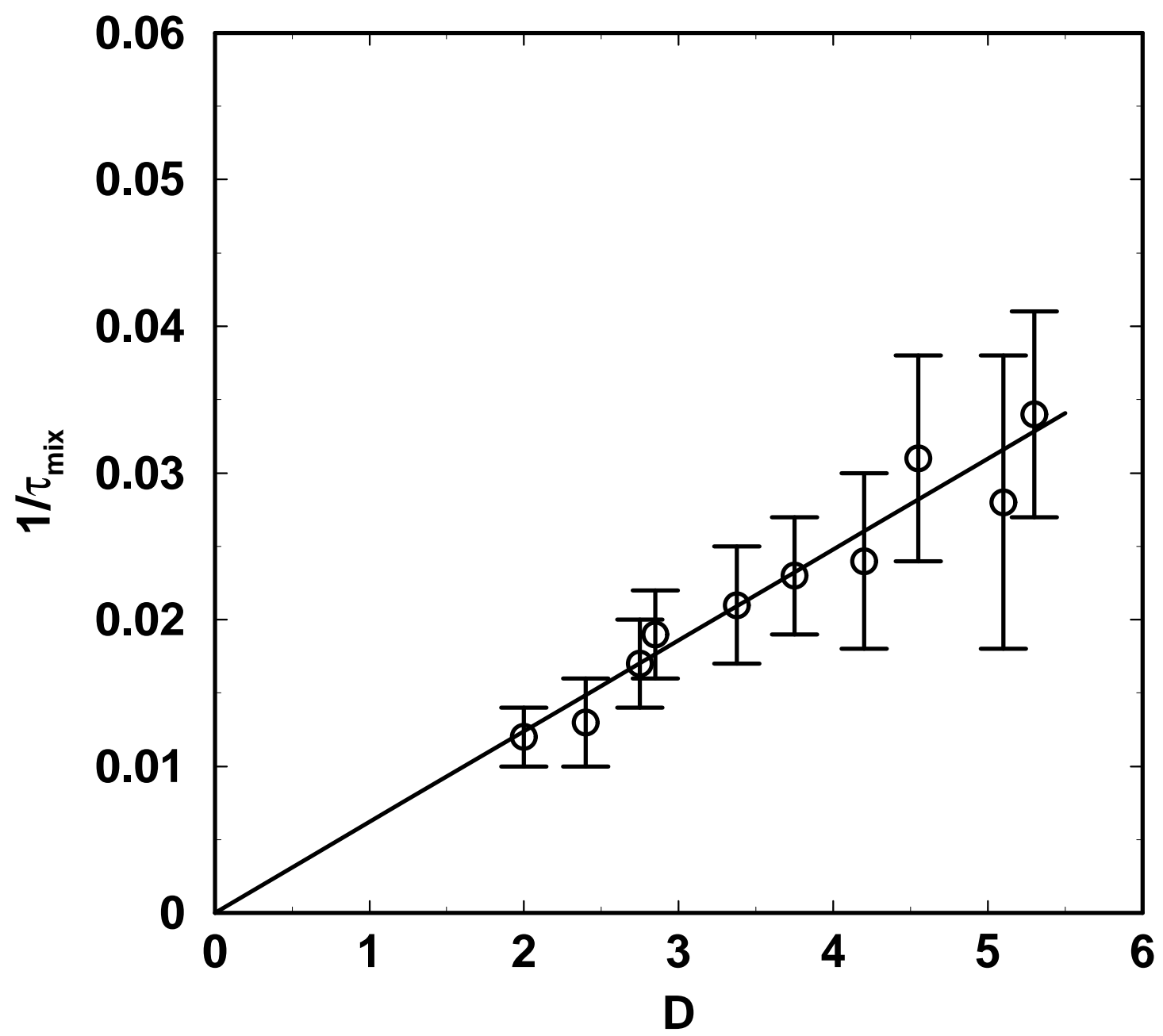

Figure 4

C. Henrique et. al.

"Diffusion as mixing process in granular materials", 

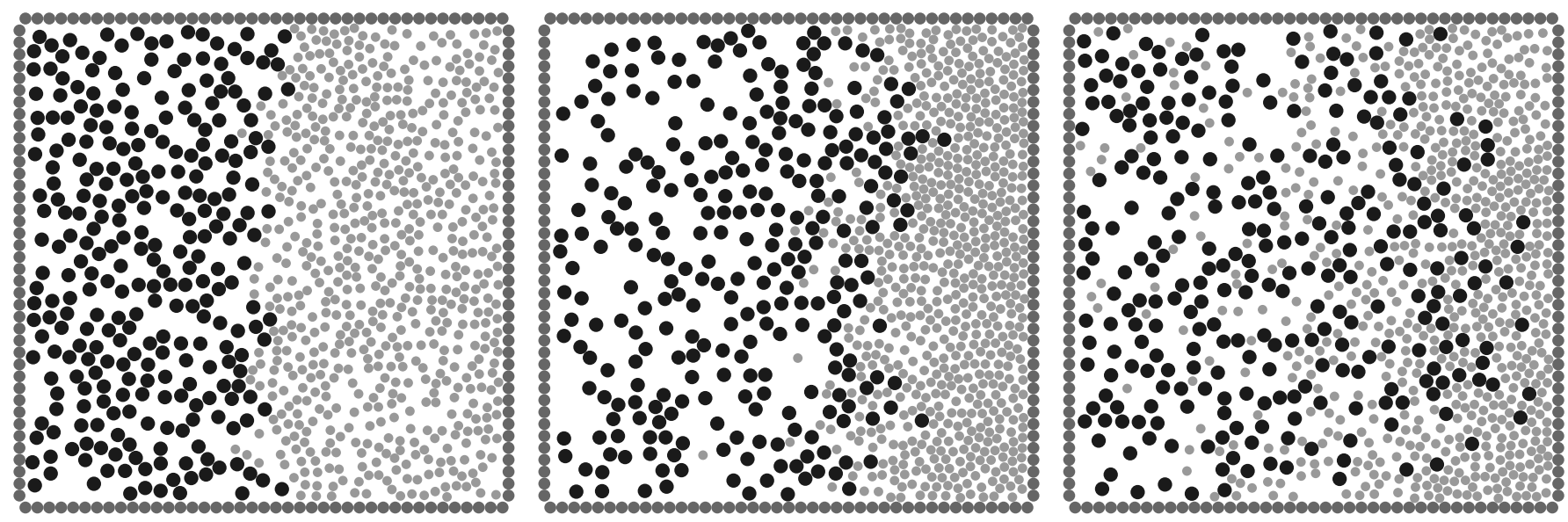

Figure 5

C. Henrique et. al.

"Diffusion as mixing process in granular materials", 


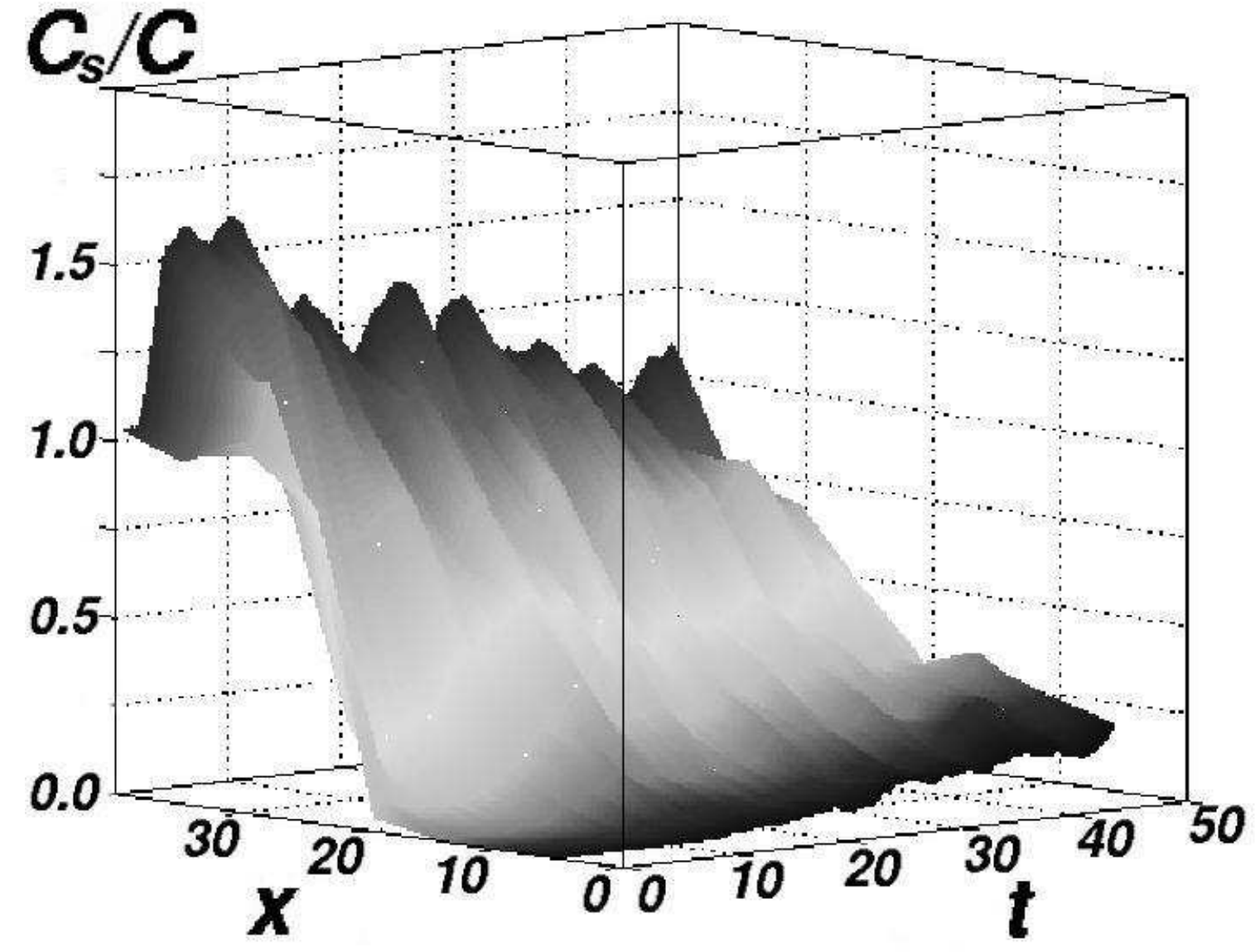

Figure 6

C. Henrique et. al.

"Diffusion as mixing process in granular materials", 


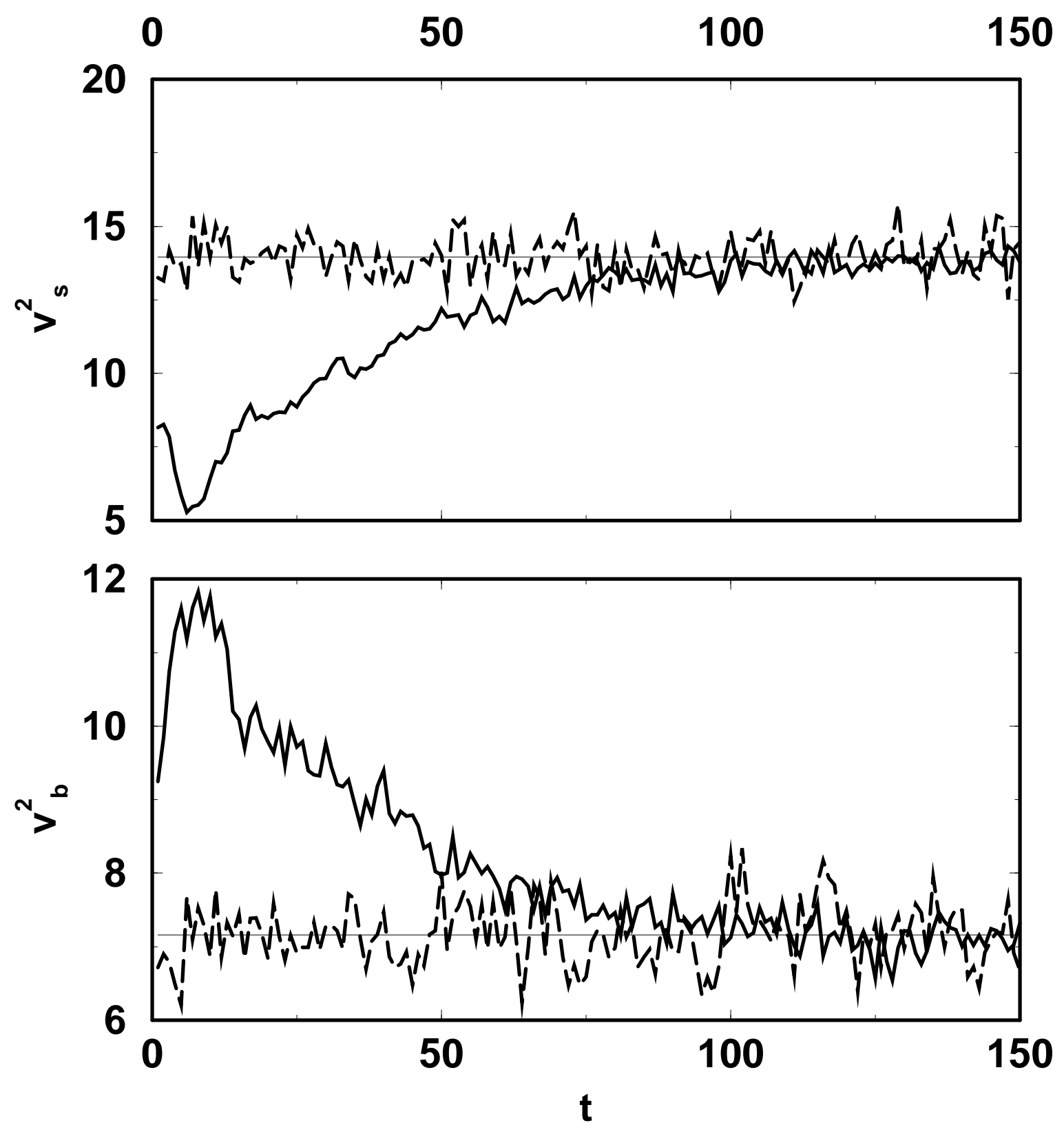

Figure 7

C. Henrique et. al.

"Diffusion as mixing process in granular materials", 


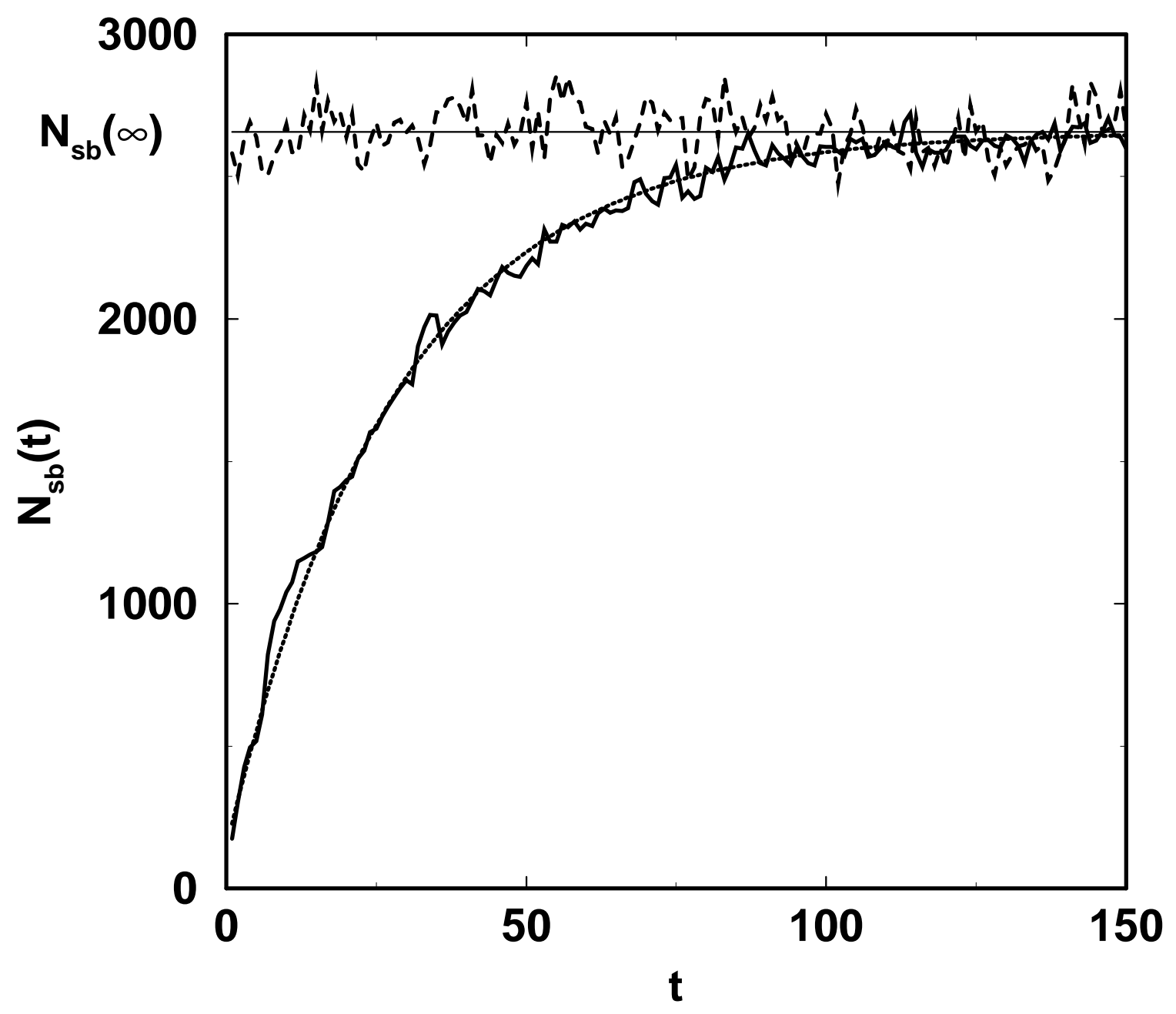

Figure 8

C. Henrique et. al.

"Diffusion as mixing process in granular materials", 


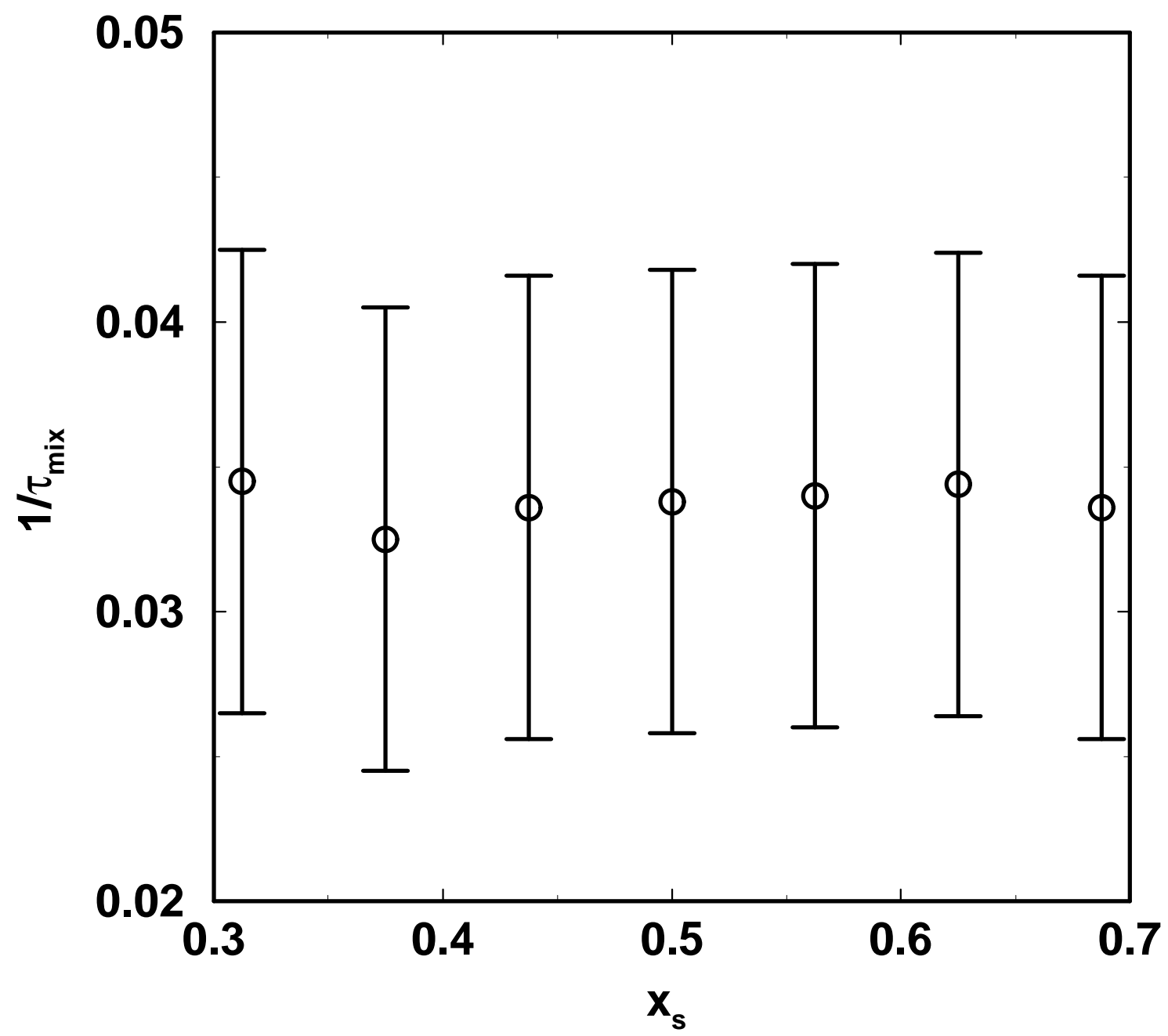

Figure 9

C. Henrique et. al.

"Diffusion as mixing process in granular materials", 


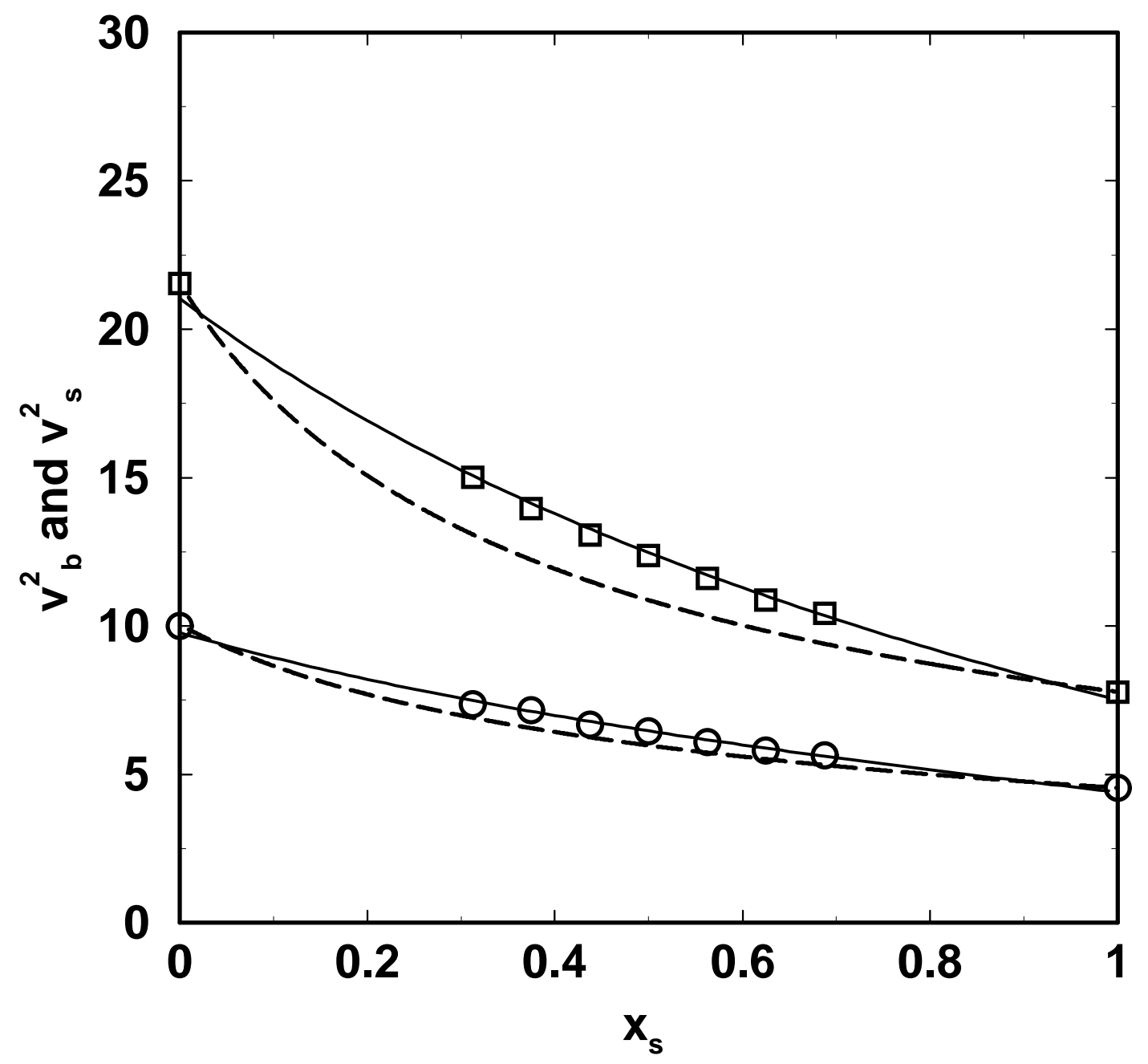

Figure 10

C. Henrique et. al.

"Diffusion as mixing process in granular materials", 


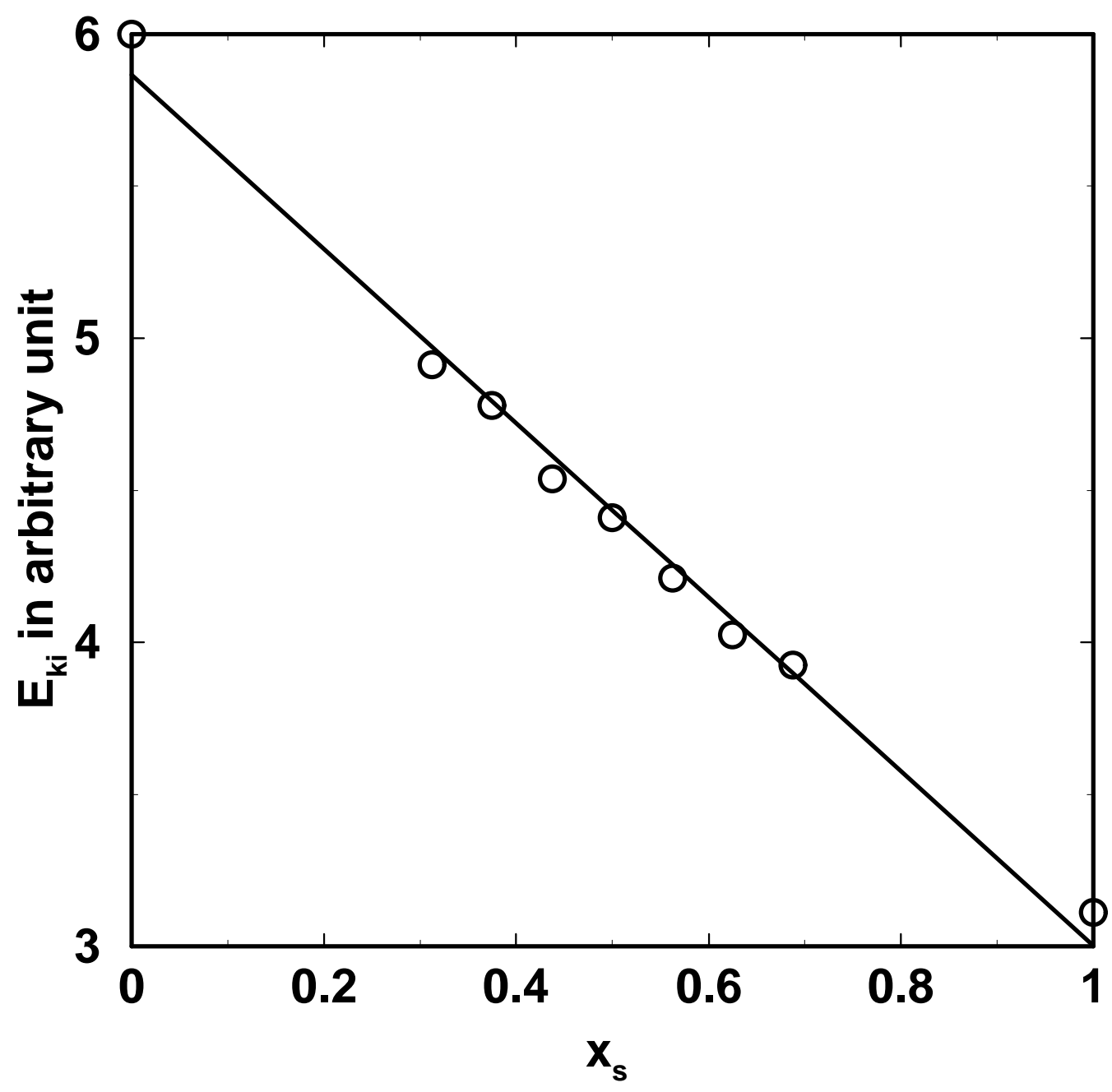

a)

Figure 11.a

C. Henrique et. al.

"Diffusion as mixing process in granular materials", 


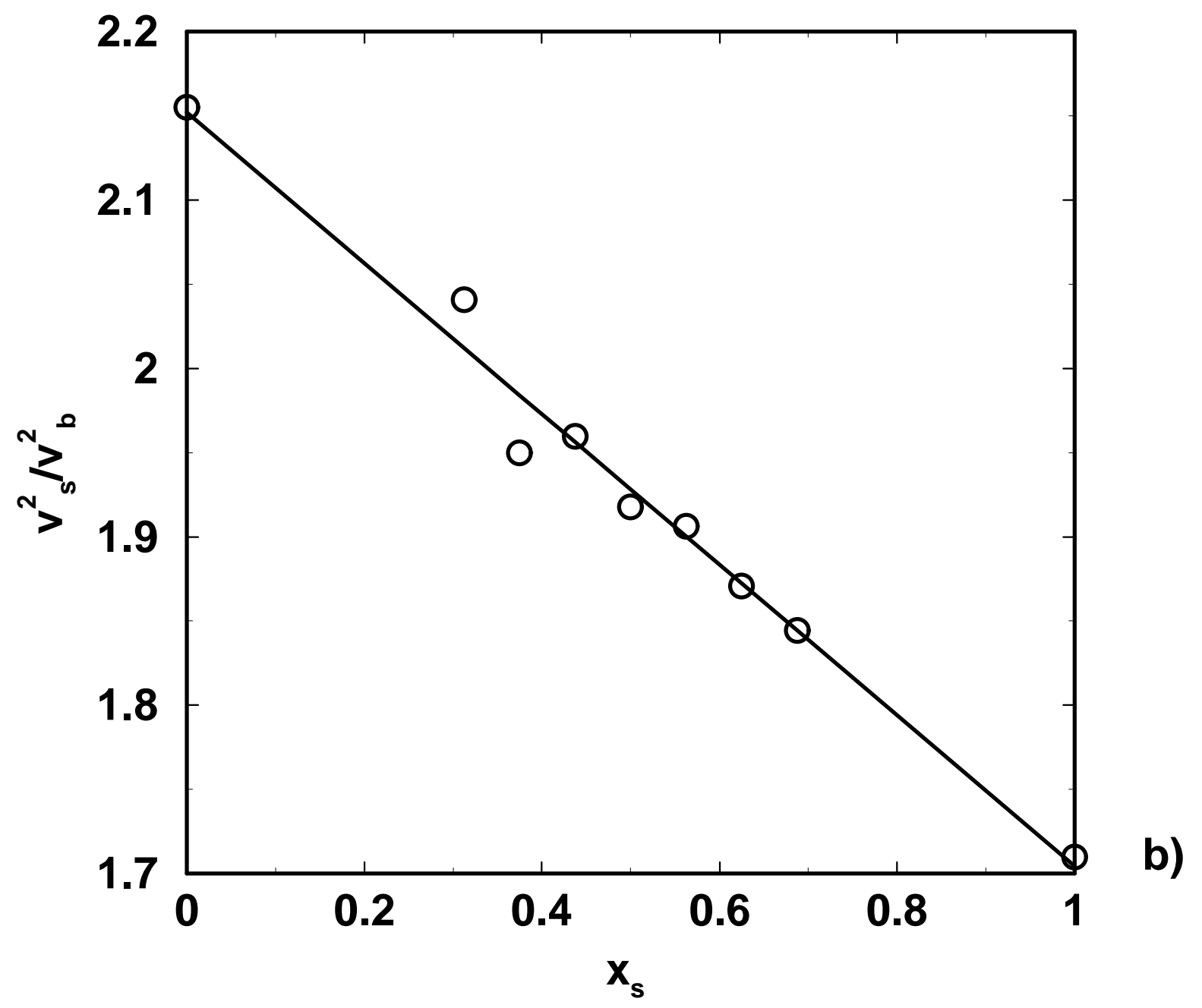

Figure 11.b

C. Henrique et. al.

"Diffusion as mixing process in granular materials" 


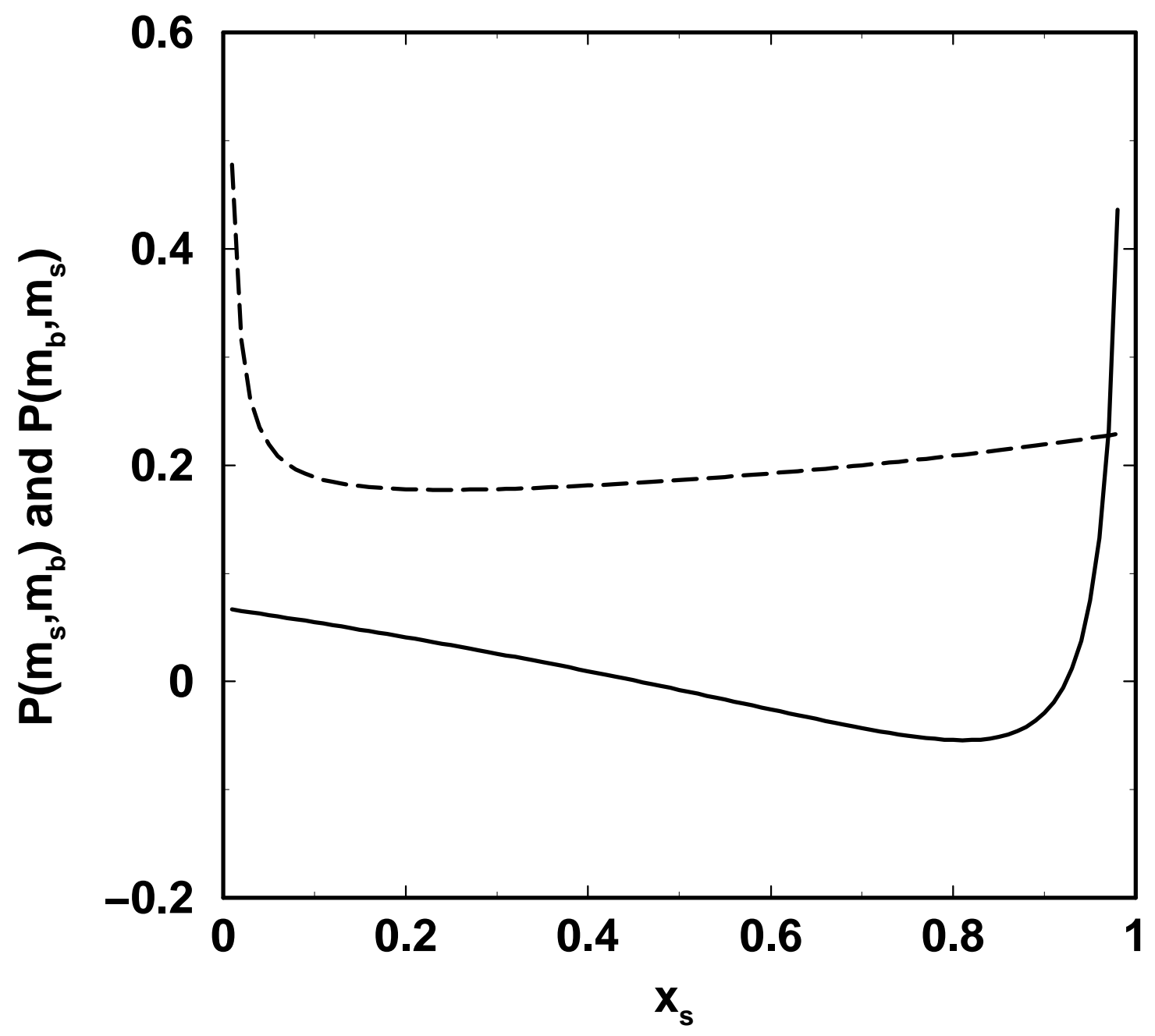

Figure 12

C. Henrique et. al.

"Diffusion as mixing process in granular materials", 


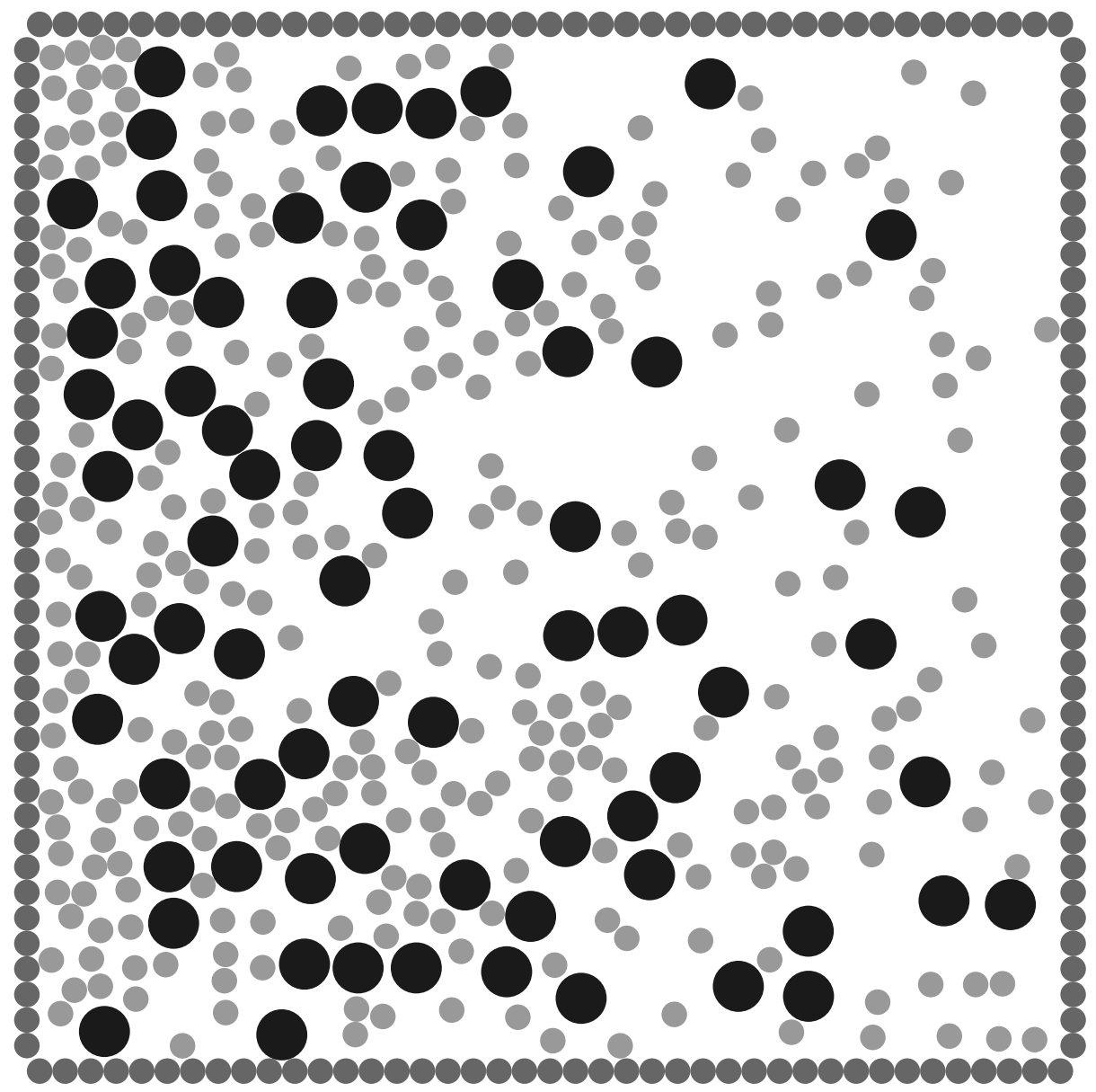

Figure 13

C. Henrique et. al.

"Diffusion as mixing process in granular materials", 


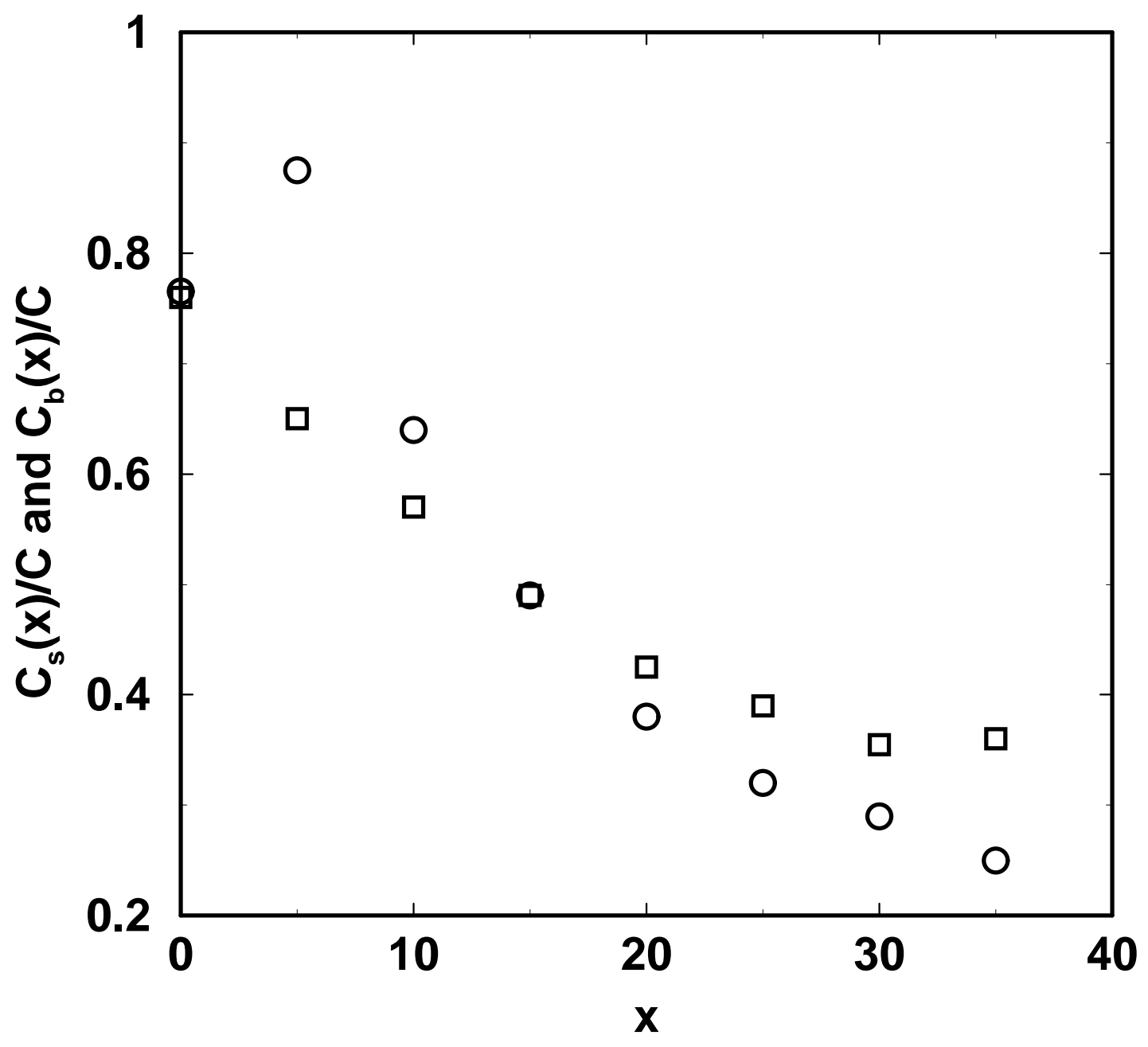

Figure 14

C. Henrique et. al.

'Diffusion as mixing process in granular materials', 